Part of Journal of Research of the National Bureau of Standards, Volume 20, May 1938

\title{
APPLICATION OF VERTICAL-INCIDENCE IONOSPHERE MEASUREMENTS TO OBLIQUE-INCIDENCE RADIO TRANSMISSION
}

\author{
By Newbern Smith
}

ABSTRACT

An extension of the transmission curves described in a previous paper is given. The effects of the earth's curvature and magnetic field are considered, as well as absorption or reflection from lower ionosphere layers. A chart is included for rapid calculation of transmission curves.

\section{CONTENTS}

I. Introduction

II. Effect of the earth's curvature

III. Effect of the earth's magnetic field

IV. Behavior of the wave below the point of reflection

V. Angle of departure and arrival of the waves_._.

VI. Determination of sec $\phi_{0}$

VII. Transmission factors

VIII. Conclusions _... 705

\section{INTRODUCTION}

In a recent number of this Journal ${ }^{1}$ the author outlined a graphic method of obtaining, from vertical-incidence ionosphere measurements, the limiting frequencies and virtual heights of reflection of radio waves incident obliquely upon the ionosphere. In that paper was developed a type of "transmission curve" which, when superimposed on a curve of frequency against virtual height, measured at vertical incidence, gave directly the virtual height of reflection for a given frequency at a given distance. A logarithmic sec $\phi_{0}$ transmission curve was also described by which, for a given distance, one curve could be used to determine the heights, to a fair approximation, at any frequency.

It is the purpose of the present paper to evaluate in more detail the effect of the earth's curvature upon the ray theory of ionosphere transmission, to indicate the modifications consequently necessitated in the application of the transmission curves, and to consider briefly how the earth's field may affect limiting frequencies and virtual heights.

\section{EFFECT OF THE EARTH'S CURVATURE}

The influence of the earth's magnetic field will be neglected in this section. In the next section the effect of the magnetic field on radio

\footnotetext{
${ }^{1}$ Newbern Smith. Extension of normal-incidence ionosphere measurements to oblique-incidence radio transmission. J. Research NBS 19, 89 (1937) RP1013.
} 
transmission will be considered for the case of the plane earth, and it will be assumed that the results hold approximately for the curved earth, also.

The elementary theory of propagation of electromagnetic waves in an ionized medium whose surfaces of equal ionic density are planes

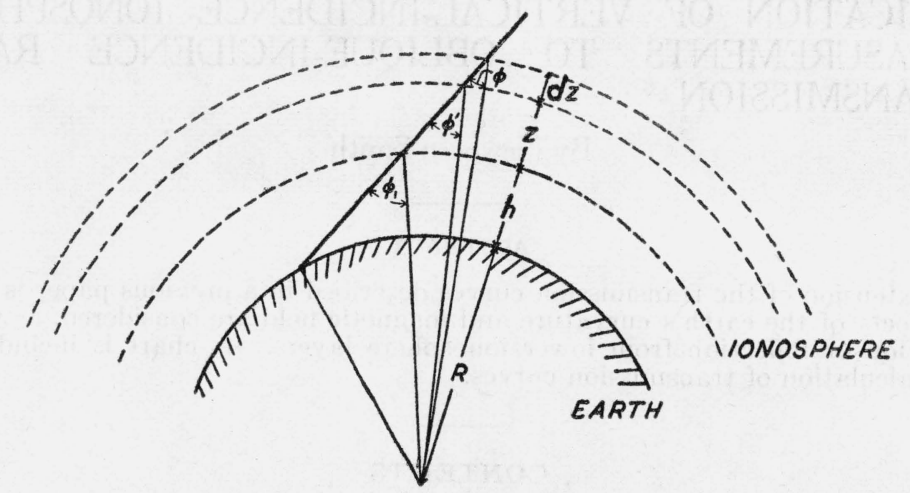

FIGURE 1.-Variation, with height, of angle a straight line makes with the normal io the earth's surface.

shows that, by Snell's law, the waves will penetrate the medium until the refractive index $\mu^{\prime}$ is reduced to the value $\sin \phi_{1}$, where $\phi_{1}$ is the angle of incidence of the waves upon the ionosphere, i. e., the angle the wave normal makes with the vertical on entering the ionosphere. To obtain the corresponding relation for a curved ionosphere it is necessary to consider the variation in the angle $\phi$ which a straight line makes with the vertical, or normal to the earth's surface, at various altitudes $(z)$.

The geometry of figure 1 leads to the relation

$$
\sin \phi^{\prime}=\sin \phi\left(1+\frac{d z}{R+h+z}\right) .
$$

Assuming Snell's law to be valid for a ray traversing an infinitely thin layer of the ionosphere, of thickness $d z$, we can use this relation to obtain the differential equation:

$$
\frac{d\left(\mu^{\prime} \sin \phi\right)}{\mu^{\prime} \sin \phi}=-\frac{d z}{R+h+z}
$$

Integrating this from the lower boundary of the ionosphere, where $\mu^{\prime}=1, \phi=\phi_{1}$, and $z=0$ (see fig. 2) up to the altitude $z$, we get

$$
\mu^{\prime} \sin \phi=\frac{\sin \phi_{1}}{1+\frac{z}{R+h}}
$$

as the form of Snell's law appropriate to the curved earth. 
This means that the wave will penetrate the curved ionosphere until the refractive index $\mu^{\prime}$ is reduced to the value $\mu_{0}{ }^{\prime}$, given by

$$
\mu_{0}^{\prime}=\frac{\sin \phi_{1}}{1+\frac{z_{0}}{R+h}},
$$

where $\phi_{1}$ is the angle of incidence of the wave on the lower boundary of the ionosphere and $z_{0}$ is the maximum height of penetration above this lower boundary.

From the geometry of figure 1, we have also

$$
\sin \phi_{1}=\sin \phi^{\prime}\left(1+\frac{z}{R+h}\right) \text {. }
$$

If we put $\phi^{\prime}=\phi_{0}$ and $z=z_{v}$, the values which these quantities have at the top of the equivalent triangular path in figure 2 , this becomes

$$
\sin \phi_{1}=\sin \phi_{0}\left(1+\frac{z_{0}}{R+h}\right)
$$

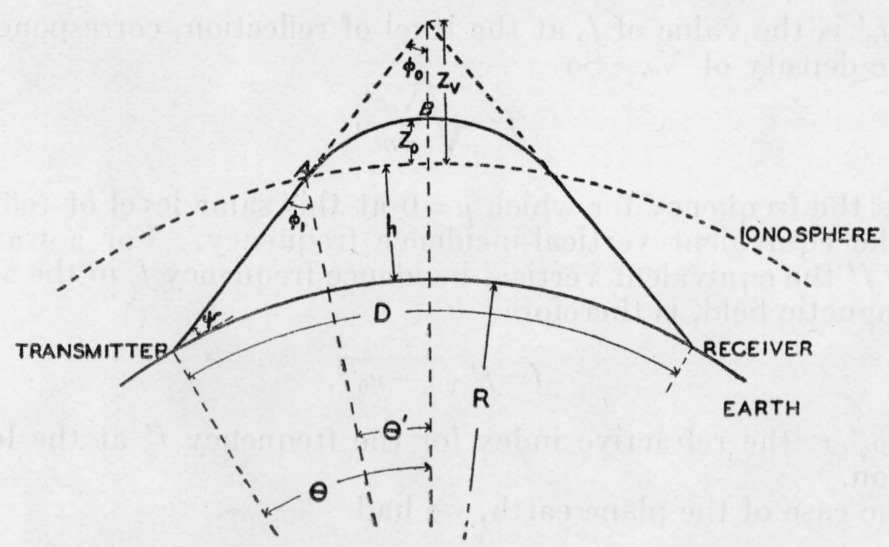

FIGURE 2.-Transmission through curved ionosphere.

$z_{0}=$ height of equivalent triangular path; $z_{0}=$ true height of reflection, at level where $\mu^{\prime}=\sin \phi_{1}\left(1-\frac{z_{0}}{R+h}\right)$, $R=$ radius of earth; and $D=$ distance of transmission.

and so the refractive index at the level of reflection must be

$$
\mu_{0}^{\prime}=\sin \phi_{0} \frac{\left(1+\frac{z_{0}}{R+h}\right)}{\left(1+\frac{z_{0}}{R+h}\right)}
$$

instead of $\mu_{0}^{\prime}=\sin \phi_{0}$, as is the case for the plane earth.

If, as is usually the case, the penetration $z_{0}<<R+h$, we may write this condition

$$
\mu_{0}^{\prime}=\sin \phi_{0}\left(1+\frac{z_{0}-z_{0}}{R+h}\right)
$$


The effect of the earth's curvature is thus to cause the radio wave to be reflected from a region of smaller ionization density than would be necessary were the earth flat.

In considering the problem of radio transmission over a distance, it will often be convenient to speak of an "equivalent vertical-incidence frequency" for the given wave frequency and transmission path. This is the frequency which is reflected at vertical incidence at the same height and undergoes approximately the same absorption as does the actual wave at oblique incidence. It will be denoted by $f$, and the actual wave frequency will be denoted by $f^{\prime}$.

Since, for a frequency $f^{\prime}$,

$$
\mu=\sqrt{1-\frac{N e^{2}}{\pi m f^{\prime 2}}}=\sqrt{1-\frac{f_{0}^{2}}{f^{\prime 2}}}
$$

where $f_{0}=\sqrt{\frac{N e^{2}}{\pi m}},{ }^{23}$ it follows that reflection of this frequency will take place at a level where

$$
\mu_{0}^{\prime}=\sqrt{1-\frac{f_{0}^{\prime 2}}{f^{\prime 2}}}
$$

where $f_{0}^{\prime}$ is the value of $f_{0}$ at the level of reflection, corresponding to an ionic density of $N_{0}$. So

$$
f=\sqrt{\frac{N_{0} e^{2}}{\pi m}}
$$

and $f$ is the frequency for which $\mu=0$ at this same level of reflection, i. e., the equivalent vertical-incidence frequency. For a wave frequency $f^{\prime}$ the equivalent vertical-incidence frequency $f$, in the absence of a magnetic field, is therefore

$$
f=f^{\prime} \sqrt{1-\mu_{0}^{\prime 2}}
$$

where $\mu_{0}^{\prime}$ is the refractive index for the frequency $f^{\prime}$ at the level of reflection.

In the case of the plane earth, we had

which gives

$$
\mu_{0}^{\prime}=\sin \phi_{1}=\sin \phi_{0},
$$

$$
f=\frac{f^{\prime}}{\sec \phi_{0}}
$$

the well-known secant law. In the case of the curved earth, however, we must use the values of $\mu_{0}^{\prime}$ given by eq 3 or eq 5 , and

$$
\sqrt{1-\mu_{0}^{\prime 2}}=\sqrt{1-\frac{\sin ^{2} \phi_{1}}{\left(1+\frac{z_{0}}{R+h}\right)^{2}}}=\sqrt{1-\sin ^{2} \phi_{0}\left(\frac{1+\frac{z_{0}}{R+h}}{1+\frac{z_{0}}{R+h}}\right)^{2}}
$$

Under practical conditions $z_{0}$ is almost always less than $400 \mathrm{~km}$, and values of $z_{v}$ greater than $600 \mathrm{~km}$ contribute nothing to trans-

2 W. H. Eccles, Proc. Roy. Soc. (London) [A] 87, 79 (1912).
3 J. Larmor, Jahrb. drahtlosen Telegraphie 25, 141 (1925). 
mission over appreciable distances, so that we can always consider $z_{0}$ and $z_{v} \ll R+h$. Using this approximation, eq 7 a becomes

$$
\sqrt{1-\mu_{0}^{\prime 2}}=\cos \phi_{1} \sqrt{1+\frac{2 z_{0}}{R+h}} \tan ^{2} \phi_{1}=\cos \phi_{0} \sqrt{1-\frac{2\left(z_{v}-z_{0}\right)}{R+h} \tan ^{2} \phi_{0}}(7 \mathrm{~b})
$$

Figure 3 gives values of $\frac{1}{\sqrt{1-\mu_{0}^{\prime 2}}}$ plotted against $\tan \phi_{1}$ for various

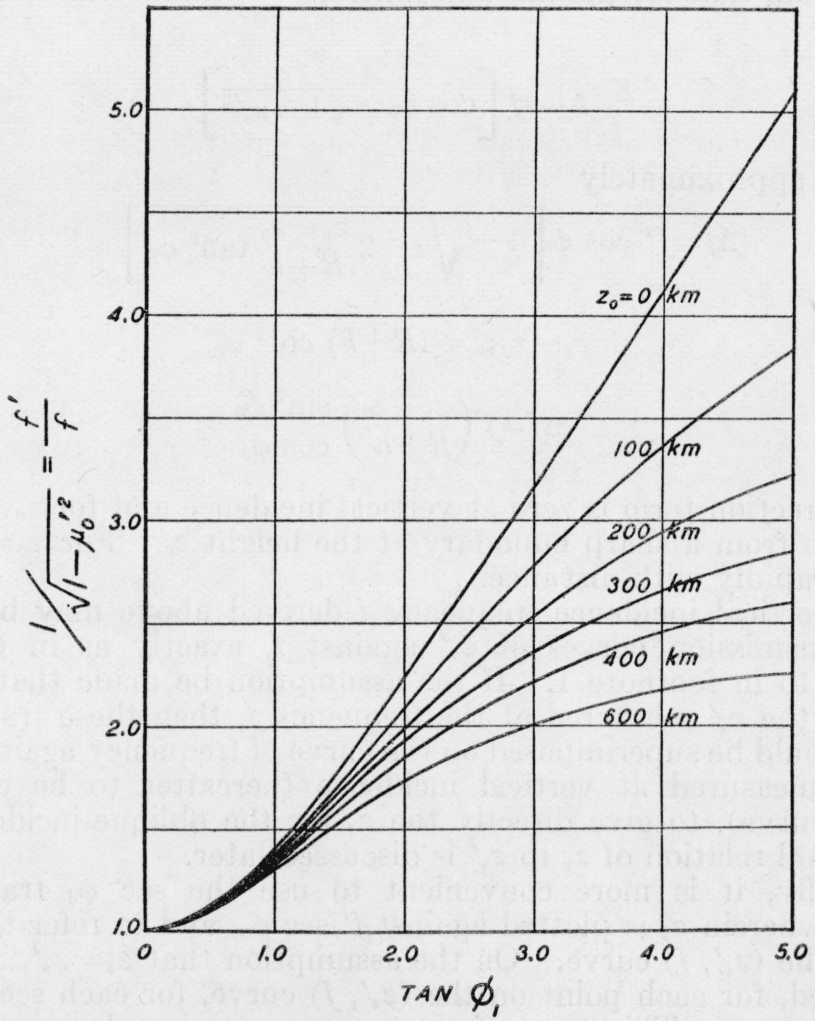

FIGURE 3.-Variation of $\frac{1}{\sqrt{1-\mu_{o}^{\prime 2}}}$ with $z_{0}$ and tan $\phi_{0}$.

values of $z_{0}$, assuming $h=100 \mathrm{~km}$ (at the bottom of the $E$ layer). These curves may be used to determine the relation of $f$ to $f^{\prime}$ for various values of $z_{0}$ and $\phi_{1}$. By substituting $\phi_{0}$ for $\phi_{1}$ and $z_{0}-z_{v}$ for $z_{0}$, similar curves may also be plotted to determine the relation of $f$ to $f^{\prime}$ for various values of $z_{0}, z_{v}$, and $\phi_{0}$.

If, further, $z_{0} \ll(R+h) \cot ^{2} \phi_{1}$ or $\left(z_{0}-z_{0}\right) \ll(R+h) \cot ^{2} \phi_{0}$ we may write eq $7 \mathrm{~b}$ approximately,

$$
\sqrt{1-\mu_{0}^{\prime 2}}=\cos \phi_{1}\left[1+\frac{z_{0}}{R+h} \tan ^{2} \phi_{1}\right]=\cos \phi_{0}\left[1-\frac{z_{v}-z_{0}}{R+h} \tan ^{2} \phi_{0}\right]
$$

a form which it is convenient to use in some discussions. This approximation leads to results good to 1 percent or better for $E$-layer 
transmission, where $z_{0}$ is less than $50 \mathrm{~km}$, and for single-reflection $F$-layer transmission over distances less than $1,500 \mathrm{~km}$, or multireflection $F$-layer transmission where each reflection covers less than $1,500 \mathrm{~km}$. For transmission over distances greater than $1,500 \mathrm{~km}$ for each reflection it is necessary to use the more exact expression.

The correction term, or frequency $\Delta f$ which it is necessary to subtract from $f^{\prime} / \mathrm{sec} \phi_{0}$ in order to obtain $f$, is the amount by which the equivalent vertical-incidence frequency for the curved ionosphere differs from that for the flat ionosphere.

This is

$$
\Delta f=f^{\prime}\left[\cos \phi_{0}-\sqrt{1-\mu_{0}^{\prime 2}}\right],
$$

which is approximately

and, if

$$
\Delta f=f^{\prime} \cos \phi_{0}\left[1-\sqrt{1-2 \frac{z_{0}-z_{0}}{R+h} \tan ^{2} \phi_{0}}\right]
$$

$$
\begin{gathered}
z_{0}-z_{0}<<(R+h) \cot ^{2} \phi_{0}, \\
\Delta f \doteq f^{\prime}\left(\frac{z_{v}-z_{0}}{R+h}\right) \frac{\sin ^{2} \phi_{0}}{\cos \phi_{0}}
\end{gathered}
$$

This correction term is zero at vertical incidence and for $z_{0}=z_{v}{ }^{\prime}$, i. e., reflection from a sharp boundary at the height $z_{0}$. For $z_{0} \neq z_{v}^{\prime}$ it increases rapidly with distance.

The vertical incidence frequency $f$ derived above may be used to plot transmission curves of $z_{v}^{\prime}$ against $f$, exactly as in the paper referred to in footnote 1 . If the assumption be made that $z_{v}$ is the same as the $z_{v}^{\prime}$ measured at the frequency $f$, then these transmission curves could be superimposed on the curve of frequency against virtual height, measured at vertical incidence (hereafter to be called the $\left(z_{v}^{\prime}, f\right)$ curve), to give directly the $z_{v}$ for the oblique-incidence case. The actual relation of $z_{v}$ to $z_{v}^{\prime}$ is discussed later.

Actually, it is more convenient to use the curves, wherein $z_{0}$ is plotted against $f^{\prime} / \mathrm{sec} \phi_{0}$, and to refer the correction to the $\left(z_{v}{ }^{\prime}, f\right)$ curve. On the assumption that $z_{v}=z_{v}{ }^{\prime}, \Delta f$ can be calculated, for each point on the $\left(z_{v}^{\prime}, f\right)$ curve, for each sec $\phi_{0}$ transmission curve. This transmission curve can then be superimposed on the $\left(z_{v}{ }^{\prime}, f\right)$ curve displaced toward the higher frequencies by the amount $\Delta f$, and the oblique-incidence values of $z_{0}$ read off.

The calculation of $\Delta f$ for each distance from $z_{v}^{\prime}$ and $z_{0}$, without any assumption as to $z_{v}$, and for a $\phi_{0}$ calculated for an equivalent triangular path of height $z_{v}^{\prime}$, is discussed below. This value of $\Delta f$ can be used in the same manner as was described in the preceding paragraph, and the sec $\phi_{0}$ curves applied, $\phi_{0}$ being calculated for an equivalent triangular path of height $z_{v}^{\prime}$ instead of $z_{v}$.

If the $\left(z_{0}^{\prime} f\right)$ curve and the sec $\phi_{0}$ curves are plotted logarithmically, as in the paper referred to, ${ }^{4}$ we may calculate a factor $1+\Delta f / f$, as follows:

$$
1+\frac{\Delta f}{f}=\frac{\cos \phi_{0}}{\sqrt{1-\mu_{0}^{\prime 2}}}
$$

Extension of normal-incidence ionosphere measurements to oblique-incidence radio transmission. J. Research NBS 19, 89 (1937) RP1013. 
The frequencies on the $\left(z_{0}{ }^{\prime}, f\right)$ curve can then be easily multiplied by this factor by adding the factor logarithmically to the curve, and the $\log$ sec $\phi_{0}$ curves can be applied to the resulting corrected $\left(z_{0}^{\prime}, f\right)$ curve. It should be noted that in this expression the $\phi_{0}$ refers only to an equivalent triangular path of height $z_{v}{ }^{\prime}$, and not $z_{0}$. The $z_{v}$ enters only in to the expression for $\mu_{0}^{\prime}$.

A typical $\left(z_{v}^{\prime}, f\right)$ curve corrected in this manner for a given distance $(2,000 \mathrm{~km})$ is shown in figure 4. The virtual heights are lower on the corrected curve and the curve extends out to frequencies higher than the critical frequency for the ordinary ray. The correction to the curve is different for different distances, increasing with the distance. This means that the virtual heights of reflection are lower, for greater distances, than if the curvature of the earth were not considered. The limiting frequency of transmission, or maximum usable frequency, over considerable distances is correspondingly increased, the difference becoming as much as 20 percent at the greater distances, depending of course on the form of the $\left(z_{v}^{\prime}, f\right)$ curve.

When the $\left(z_{v}^{\prime}, f\right)$ curve has been corrected in the above manner it may be plotted logarithmically, as described in the previous paper mentioned above, and the logarithmic sec $\phi_{0}$ curves may be applied to give directly the maximum usable frequencies and virtual heights over transmission pathsof givenlengths.

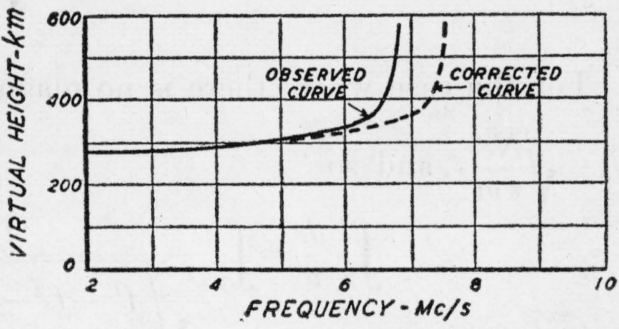

FIGURE 4.- $\left(z_{v}^{\prime}, f\right)$ curve corrected for a given distance $(2,000 \mathrm{~km})$ for the effect of the earth's curvature.

Since the first draft of this paper was written there has come to the author's attention an excellent unpublished paper "Skip-Distance Analysis," by T. L. Eckersley and G. Millington, in the form of a contribution to the November 1937 London meeting of the Special Radio Wave Propagation Committee held in preparation for the Cairo Radio Conference. In this they undertake an analysis of radio transmission over a curved earth and obtain curves for determining the retardation of sky wave over ground wave at a distance, in terms of vertical-incidence measurements. They limit the analysis, however, to the case where $z_{0}$ is very small compared with $h$. The analysis can thus apply only to $E$-layer transmission, since for $F$-layer transmission, $z_{0}$ must be measured from the lower boundary of the ionosphere in order to include the effect of retardation in the $E$ layer.

Their work, however, suggests a method of approximate analysis for larger values of $z_{0}$ and a means for easily estimating the error involved in the assumption made above that the $z_{0}$ for a given frequency $f^{\prime}$ was equal to the vertical-incidence $z_{v}^{\prime}$ for the equivalent vertical-incidence frequency $f$.

The development given by Eckersley and Millington begins with the following relation, for the ray path shown in figure 2:

$$
\theta^{\prime}=\int_{A}^{B} d \theta=\int_{A}^{B} \frac{d s \sin \phi}{R+h+z},
$$


where $d s$ is an element of the ray path making an angle with the vertical. Since, from eq 2

this becomes

$$
\sin \phi=\frac{1}{\mu^{\prime}} \frac{\sin \phi_{1}}{1+\frac{z}{R+h}},
$$

They also used the relation

$$
\theta^{\prime}=\frac{\sin \phi_{1}}{R+h} \int_{A}^{B} \frac{d s}{\mu^{\prime}\left(1+\frac{z}{R+h}\right)^{2}}
$$

$$
\int_{A}^{B} \frac{d s}{\mu^{\prime}}=\int_{0}^{z_{0}} \frac{d z}{\mu^{\prime} \cos \phi}=\int_{0}^{z_{0}} \frac{d z}{\sqrt{\mu^{\prime 2}-\frac{\sin ^{2} \phi_{1}}{\left(1+\frac{z}{R+h}\right)^{2}}}} .
$$

For the case where there is no magnetic field $\mu^{\prime}=\sqrt{1-\frac{f_{0}^{2}}{f^{\prime 2}}}$, where $f_{0}=\sqrt{\frac{N_{e}^{2}}{\pi m}}$, and so

$$
\int_{A}^{B} \frac{d s}{\mu^{\prime}}=\int_{0}^{z_{0}} \frac{f^{\prime} d z}{\sqrt{f^{\prime 2}-f_{0}^{2}-f^{\prime 2} \frac{\sin ^{2} \phi_{1}}{\left(1+\frac{z}{R+h}\right)^{2}}}} .
$$

Putting into this expression the wave frequency $f^{\prime}$ in terms of the equivalent vertical-incidence frequency given above in eq 6 .

$$
\int_{A}^{B} \frac{d s}{\mu^{\prime}}=\frac{1}{\sqrt{1-\mu_{0}^{\prime 2}}} \int_{0}^{z_{0}} \frac{f d z}{\sqrt{\frac{f^{2}}{1-\mu_{0}^{\prime 2}}\left(1-\frac{\sin ^{2} \phi_{1}}{\left(1+\frac{z}{R+h}\right)^{2}}\right)-f_{0}^{2}}}
$$

At this point Eckersley and Millington assumed that they were dealing with a thin layer, and that in consequence (1) $\theta^{\prime}$ was only slightly less than $\frac{\sin \phi_{1}}{R+h} \int_{A}^{B} \frac{d s}{\overline{\mu^{\prime}}}$, from eq $8,(2) \int_{A}^{B} \frac{d s}{\overline{\mu^{\prime}}}$ was only slightly greater than $\frac{1}{\sqrt{1-\mu_{0}^{\prime 2}}} \int_{0}^{z_{0}} \frac{f d z}{\sqrt{f^{2}-f_{0}^{2}}}$, from eq 11. This simplification is justified for $E$-layer transmission, but is unfortunately not justified for $F_{2}$-layer transmission, since the lower boundary of the ionosphere must be taken at the beginning of the E-layer in each case. We shall not, therefore, confine our discussion to this limited case. It must also be noted that the simplification introduced by assuming $z_{0}<<$ $(R+h) \cot ^{2} \phi_{1}$ also is not valid except at comparatively short distances, 
where $\phi_{1}=45^{\circ}$ or less. For practical purposes, it is, however, justifiable to assume $z_{0}<<R+h$. Using this relation then, we obtain

$$
\theta^{\prime}=\frac{\sin \phi_{1}}{R+h} \int_{A}^{B} \frac{d s}{\mu^{\prime}}\left(1-\frac{2 z}{R+h}\right)
$$

Eckersley and Millington combined the approximate forms of eq 8 and 11 and introduced the value of $\sqrt{1-\mu_{0}^{\prime 2}}=\cos \phi_{1}$, to obtain $\theta^{\prime}=\frac{\tan \phi_{1}}{R+h} \int_{0}^{z_{0}} \frac{f d z}{\sqrt{f^{2}-f_{0}^{2}}} \cdot$ We shall, however, combine eq 11 and 12 to obtain the more exact relation

$$
\theta^{\prime}=\frac{\sin \phi_{1}}{(R+h) \sqrt{1-\mu_{0}^{\prime 2}}} \int_{0}^{z_{0}} \frac{f d z}{\sqrt{f^{2}(1-A)-f_{0}^{2}}}\left(1-\frac{2 z}{R+h}\right)
$$

where

$$
1-A=\frac{1-\frac{\sin ^{2} \phi_{1}}{\left(1+\frac{z}{R+h}\right)^{2}}}{1-\mu_{0}^{\prime 2}}=\frac{1+\frac{2 z}{R+h} \tan ^{2} \phi_{1}}{1+\frac{2 z_{0}}{R+h} \tan ^{2} \phi_{1}}
$$

If we let

$$
\begin{gathered}
1+B=\frac{1}{\sqrt{1-A\left(\frac{f^{2}}{f^{2}-f_{0}^{2}}\right)}}, \\
\theta^{\prime}=\frac{\sin \phi_{1}}{(R+h) \sqrt{1-\mu_{0}^{\prime 2}}} \int_{0}^{z_{0}} \frac{d z}{\sqrt{1-\frac{f_{0}^{2}}{f^{2}}}}(1+B)\left(1-\frac{2 z}{R+h}\right)
\end{gathered}
$$

For values of $z_{0}$ considerably less than $(R+h) \cot ^{2} \phi_{1} B$ is usually a small number, and for $z \rightarrow z_{0}$ (near the level of reflection) it is also usually small. It may attain, however, relatively large values.

In general, the evaluation of $B$ involves a knowledge of the distribution of ionic density, and a precise evaluation of the quantity $\theta^{\prime}$ involves graphic integration for each case considered. We shall for the present consider two principal cases, $z_{0} \rightarrow 0$ and $z_{0}=z_{v}{ }^{\prime}$, the virtual height at vertical incidence for the frequency $f=f^{\prime} \sqrt{1-\mu_{0}^{\prime 2}}$, and discuss some cases of linear distributions of ionic density, where $z / z_{0}=f_{0}^{2} / f^{2}$.

(a) $z_{0} \ll(R+h) \cot ^{2} \phi_{1}$. This case applies to $E$-layer transmission at any distance or to $F$-layer transmission at short distances $\left(\phi_{1}\right.$ small).

Combining eq 6 and 7 we get

$$
f=f^{\prime} \cos \phi_{1}\left[1+\frac{z_{0}}{R+h} \tan ^{2} \phi_{1}\right]
$$

and from eq $12 \mathrm{~b}$

$$
1-A=1+2\left(\frac{z-z_{0}}{R+h}\right) \tan ^{2} \phi_{1}
$$


From eq 12c

$$
1+B=1+\left(\frac{z_{0}}{R+h}\right) \frac{1-\frac{z}{z_{0}}}{1-\frac{f_{0}^{2}}{f^{2}}} \tan ^{2} \phi_{1}
$$

if we assume that $\left(1-z / z_{0}\right) /\left(1-f_{0}{ }^{2} / f^{2}\right)$ is nowhere so large as to make $B$ comparable with unity. This is equivalent to assuming that $f_{0}{ }^{2} / f^{2}$ does not approach 1 much more rapidly than does $z / z_{0}$, an assumption that is valid except quite close to a critical frequency. Transmissions involving equivalent vertical-incidence frequencies close to the critical frequency are of interest, however, only when the distance of transmission is short, and in this case $\phi_{1}$ is small, so that $B$ is a small number, anyhow.

Thus

$$
\begin{gathered}
\theta^{\prime}=\frac{\tan \phi_{1}}{R+h}\left[1-\frac{z_{0}}{R+h} \tan ^{2} \phi_{1}\right] \int_{0}^{z_{0}} \frac{d z}{\sqrt{1-\frac{f_{0}^{2}}{f^{2}}}} \\
\left\{1+\frac{z_{0}}{R+h} \tan ^{2} \phi_{1} \frac{\left(1-\frac{z}{z_{0}}\right)}{\left(1-\frac{f_{0}^{2}}{f^{2}}\right)}-\frac{2 z}{R+h}\right\} .
\end{gathered}
$$

Now $\int_{0}^{z_{0}} \frac{d z}{\sqrt{1-\frac{f_{0}^{2}}{f^{2}}}}=z_{0}^{\prime}$, the virtual height measured at vertical inci-

dence for the equivalent vertical-incidence frequency $f$. Since the terms involving $z_{0} / R+h$ are small, we may write

where

$$
\theta^{\prime}=\frac{z_{v}^{\prime} \tan \phi_{1}}{R+h}\left[1-\frac{z_{0}}{R+h} \tan ^{2} \phi_{1}(1-C)-C^{\prime}\right],
$$

$$
\begin{gathered}
C=\frac{1}{z_{v}^{\prime}} \int_{0}^{z_{0}} \frac{d z}{\sqrt{1-\frac{f_{0}^{2}}{f^{2}}}}\left(\frac{1-\frac{z}{z_{0}}}{1-\frac{f_{0}^{2}}{f^{2}}}\right), \text { and } \\
C^{\prime}=\frac{2}{z_{0}^{\prime}(R+h)} \int_{0}^{z_{0}} \frac{z d z}{\sqrt{1-\frac{f_{0}^{2}}{f^{2}}}}
\end{gathered}
$$

For a linear distribution of ionic density

$$
\begin{aligned}
1-\frac{z}{z_{0}} & =1-\frac{f_{0}^{2}}{f^{2}}, z_{0}{ }^{\prime}=2 z_{0}, \text { and } C=1, \text { and } \\
\theta^{\prime} & =\frac{\tan \phi_{1}}{R+h} \int_{0}^{z_{0}} \frac{d z}{\sqrt{1-\frac{z}{z_{0}}}}\left[1-\frac{2 z}{R+h}\right], \\
& =\frac{z_{v}{ }^{\prime} \tan \phi_{1}}{R+h}\left[1-\frac{2}{3}\left(\frac{z_{0}^{\prime}}{R+h}\right)\right] .
\end{aligned}
$$


For $z_{0}$ vanishingly small $C^{\prime} \rightarrow 0$ and eq 14 reduces simply to

$$
\theta^{\prime}=\frac{z_{v}^{\prime} \tan \phi_{1}}{R+h}
$$

(b) $z_{0} \rightarrow z_{v}{ }^{\prime}$. This case applies to reflection from a fairly sharp boundary at the level $z_{0}$, the refractive index being nearly unity up to nearly this level. An example of this would be reflection from the sporadic $\mathrm{E}$ region. The angular distance of the part of the ray path where $\mu$ departs appreciably from unity is small and so the ionosphere can be considered as essentially flat. This is the condition assumed by the author in the paper already referred to (RP1013). Here $z_{0}=z_{0}^{\prime}$ and

In this case

$$
\tan \phi_{0}=\frac{\sin \theta^{\prime}}{\frac{z_{v}^{\prime}}{R+h}+1-\cos \theta^{\prime}} .
$$

$$
\sqrt{1-\mu_{0}^{\prime 2}}=\cos \phi_{0}\left(1-\frac{z_{v}{ }^{\prime}-z_{0}}{R+h} \tan ^{2} \phi_{0}\right)
$$

For $z_{v}^{\prime}-z_{0}$ vanishingly small compared with $(R+h) \cot ^{2} \phi_{0}$, this reduces to the case for the plane ionosphere, where

$$
\sqrt{1-\mu_{0}^{\prime 2}}=\cos \phi_{0} \text {. }
$$

(c) $z_{0}=\frac{1}{2} z_{v}{ }^{\prime}$, i. e., a linear distribution of ionic density. Here $1-f_{0}^{2} / f^{2}=1-z / z_{0}$ and eq $12 \mathrm{a}$ becomes

$$
\theta^{\prime}=\frac{\sin \phi_{1}}{(R+h) \sqrt{1-\mu_{0}^{\prime 2}}} \int_{0}^{z_{0}} \frac{d z\left(1-\frac{2 z}{R+h}\right)}{\sqrt{1-A-\frac{z}{z_{0}}}}
$$

Putting in the value of $1-A$ and integrating

$$
\theta^{\prime}=z_{v}^{\prime} \frac{\sin \phi_{1} \sqrt{1+\frac{2 z_{0}}{R+h} \tan ^{2} \phi_{1}}}{(R+h) \sqrt{1-\mu_{0}^{\prime 2}}}\left(1-\frac{2}{3} \frac{z_{\imath}^{\prime}}{R+h}\right) .
$$

Now since we may consider $z_{0} \ll R+h$ we can write, as in eq $7 \mathrm{~b}$

$$
\sqrt{1-\mu_{0}^{\prime 2}}=\cos \phi_{1} \sqrt{1+\frac{2 z_{0}}{R+h} \tan ^{2} \phi_{1}}
$$

and thus eq 17 becomes

$$
\theta^{\prime}=\frac{z_{v}^{\prime} \tan \phi_{1}}{R+h}\left[1-\frac{2}{3} \frac{z_{v}^{\prime}}{R+h}\right]
$$

just as in the case where $z_{0} \ll(R+h) \cot ^{2} \phi_{1}$. We must, however, write for the equivalent vertical-incidence frequency in this case

$$
f=f^{\prime} \cos \phi_{1} \sqrt{1+\frac{2 z_{0}}{R+h} \tan ^{2} \phi_{1}}
$$


instead of the value

$$
f=f^{\prime} \cos \phi_{1}\left[1+\frac{z_{0}}{R+h} \tan ^{2} \phi_{1}\right]
$$

which we could use when $z_{0} \ll(R+h) \cot ^{2} \phi_{1}$.

The three examples just treated give an idea of what happens on transmission through the ionosphere, and of the angular distance $\theta^{\prime}$ the wave travels in the ionosphere as a function of angle of incidence $\phi_{1}$ (or vertex angle of equivalent triangular path $\phi_{0}$ ), of true height of reflection $z_{0}$ and of virtual height measured at vertical incidence $z_{v}^{\prime}$. We must now consider the part of the path from the earth to the lower boundary of the ionosphere. If we consider the incident ray to traverse an angular distance $\left(\theta-\theta^{\prime}\right)$ in going from the earth to the lower boundary of the ionosphere, the geometry of figure 2 tells us that

$$
\tan \phi_{1}=\frac{\sin \left(\theta-\theta^{\prime}\right)}{\frac{h}{R}+1-\cos \left(\theta-\theta^{\prime}\right)}
$$

For a given $\phi_{1}$, then, we may solve this equation for $\left(\theta-\theta^{\prime}\right)$, and, by adding this angle to the angle $\theta^{\prime}$ already computed, we obtain the entire angular distance $\theta$ traversed by the wave from the ground to the point of reflection in the ionosphere. For $h=100 \mathrm{~km}$, as we are assuming, it is sufficiently accurate to replace $\sin \left(\theta-\theta^{\prime}\right)$ by $\left(\theta-\theta^{\prime}\right)$ and $1-\cos \left(\theta-\theta^{\prime}\right)$ by $\frac{1}{2}\left(\theta-\theta^{\prime}\right)^{2}$ in this equation. We can thus solve for $\left(\theta-\theta^{\prime}\right)$, obtaining

$$
\theta-\theta^{\prime}=\cot \phi_{1}-\sqrt{\cot ^{2} \phi_{1}-\frac{2 h}{R}}
$$

We can now write the expression for the total distance of transmission $D$ in terms of $\phi_{1}$ (or $\left.\phi_{0}\right) z_{0}$ and $z_{0}{ }^{\prime}$ :

$$
D=2 R\left(\cot \phi_{1}-\sqrt{\left.\cot ^{2} \phi_{1}-\frac{2 h}{R}\right)}+\theta^{\prime}\right.
$$

where $\theta^{\prime}$ is computed as above, from eq $12 \mathrm{a}$.

The time $T$ required for the sky wave to travel from the transmitter to the receiver is, if $c=$ velocity of the wave in vacuum,

$$
T=\frac{2}{c}\left(R \frac{\sin \left(\theta-\theta^{\prime}\right)}{\sin \phi_{1}}+\int_{\Delta}^{B} \frac{d s}{\mu}\right)
$$

If we put in the value of $\int_{A}^{B} d s / \mu$ obtained on the basis of the above analysis, and replace $\sin \left(\theta-\theta^{\prime}\right)$ by its value in terms of $\phi_{1}$, we may express $T$ in terms of the quantities $\phi_{1}\left(\right.$ or $\left.\phi_{0}\right), z_{0}$ and $z_{v}{ }^{\prime}$, as was done with $D$.

The relation between the height $z_{0}$ of the equivalent triangular path and the virtual height $z_{v}^{\prime}$ measured at vertical incidence for the 
equivalent vertical-incidence frequency $f=f^{\prime} \sqrt{1-\mu_{0}^{\prime 2}}$ can now be written. From the geometry of figure 2,

so that

$$
\tan \phi_{0}=\tan \left(\phi_{1}-\theta^{\prime}\right)=\frac{\sin \theta^{\prime}}{\frac{z_{0}}{R+h}+1-\cos \theta^{\prime}},
$$

$$
z_{v}=(R+h)\left[\sin \theta^{\prime} \cot \left(\phi_{1}-\theta^{\prime}\right)-1+\cos \theta^{\prime}\right]
$$

The relation between $z_{v}, z_{v}{ }^{\prime}, z_{0}, \phi_{1}, \phi_{0}, \theta^{\prime}, D, f^{\prime}$, and $f$ may be summarized for the cases discussed here.

(a) $z_{0}<<\frac{1}{2}(R+h) \cot ^{2} \phi_{1}$

$$
\begin{gathered}
f=f^{\prime} \cos \phi_{1}\left[1+\frac{z_{0}}{R+h} \tan ^{2} \phi_{1}\right], \\
\theta^{\prime}=\frac{\tan \phi_{1}}{R+h}\left[1-\frac{z_{0}}{R+h} \tan ^{2} \phi_{1}\right] \int_{0}^{z_{0}} \frac{d z}{\sqrt{1-\frac{f_{0}^{2}}{f^{2}}}} \\
\left.1+\frac{z_{0}}{R+h} \tan ^{2} \phi_{1}\left(\frac{1-\frac{z}{z_{0}}}{1-\frac{f_{0}^{2}}{f^{2}}}\right)-\frac{2 z}{R+h}\right\}
\end{gathered}
$$

$T=\frac{2}{c}\left[\frac{R\left(\theta-\theta^{\prime}\right)}{\sin \phi_{1}}+\frac{1}{\cos \phi_{1}} \int_{0}^{z_{0}} \frac{d z}{\sqrt{1-\frac{f_{0}^{2}}{f^{2}}}}\left(1-\frac{z_{0}}{R+h} \tan ^{2} \phi_{1}\left(1-\frac{1-\frac{z}{z_{0}}}{1-\frac{f_{0}^{2}}{f^{2}}}\right)\right)\right]$,

$$
\begin{aligned}
\theta-\theta^{\prime} & =\cot \phi_{1}-\sqrt{\cot ^{2} \phi_{1}-\frac{2 h}{R}}, \\
D & =2 R \theta \\
z_{v} & =(R+h)\left[\sin \theta^{\prime} \cot \left(\phi_{1}-\theta^{\prime}\right)-1+\cos \theta^{\prime}\right], \\
z_{v}^{\prime} & =\int_{0}^{z_{0}} \frac{d z}{\sqrt{1-\frac{f_{0}^{2}}{f^{2}}}},
\end{aligned}
$$

$\left(a^{\prime}\right) z_{0}=0$

$$
\begin{aligned}
f & =f^{\prime} \cos \phi_{1}, \\
\theta^{\prime} & =\frac{z_{v}^{\prime} \tan \phi_{1}}{R+h}, \\
T & =\frac{2}{c}\left[\frac{R\left(\theta-\theta^{\prime}\right)}{\sin \phi_{1}}+\frac{z_{v}^{\prime}}{\cos \phi_{1}}\right], \\
\theta-\theta^{\prime} & =\cot \phi_{1}-\sqrt{\cot ^{2} \phi_{1}-\frac{2 h}{R}}, \\
D & =2 R \theta, \text { and } \\
z_{v} & =(R+h)\left[\sin \theta^{\prime} \cot \left(\phi_{1}-\theta^{\prime}\right)-1+\cos \theta^{\prime}\right],
\end{aligned}
$$


(b) linear gradient of ionic density $\left(z_{0}=\frac{1}{2} z_{v}^{\prime}\right)$

(c) $z_{0}=z_{0}^{\prime}$,

$$
\begin{aligned}
f & =f^{\prime} \cos \phi_{1} \sqrt{1+\frac{z_{v}^{\prime}}{R+h} \tan ^{2} \phi_{1},} \\
\theta^{\prime} & =\frac{z_{v}^{\prime} \tan \phi_{1}}{R+h}\left[1-\frac{2}{3} \frac{z_{0}^{\prime}}{R+h}\right], \\
T & =\frac{2}{c}\left[\frac{R\left(\theta-\theta^{\prime}\right)}{\sin \phi_{1}}+\frac{z_{v}^{\prime}}{R+h}\right], \\
\theta-\theta^{\prime} & =\cot \phi_{1}-\sqrt{\cot ^{2} \phi_{1}-\frac{2 h}{R},} \\
D & =2 R \theta, \\
z_{v} & =(R+h)\left[\sin \theta^{\prime} \cot \left(\phi_{1}-\theta^{\prime}\right)-1+\cos \theta^{\prime}\right], \\
f & =f^{\prime} \cos \phi_{0}, \\
\tan \phi_{0}= & \frac{\sin \theta^{\prime}}{R+h}=\frac{\sin \theta}{R+1-\cos \theta^{\prime}} \frac{z_{v}^{\prime}+h}{R}+1-\cos \theta \\
T & =\frac{2}{c} \frac{(R+h) \sin \theta}{\sin \phi_{0}} \\
D= & 2 R \theta, \text { and } \\
z_{0}= & z_{v}^{\prime}
\end{aligned}
$$

Case $\left(a^{\prime}\right)$ corresponds to the special case treated by Eckersley and Millington, except that, for simplicity of analytical computation, they took $R+h=R$ in the expression for $\theta^{\prime}$, which introduces an error of about $0.016 h$ percent ( $h$ expressed in $\mathrm{km}$ ) - not a serious error for $h=100 \mathrm{~km}$, as we have assumed. This error becomes appreciable, however, if it is attempted to extend this treatment, as they have done, to a height of $400 \mathrm{~km}$ or so.

Martyn's equivalence theorem, ${ }^{5}$ developed for a plane ionosphere, tells us that

where

$$
\begin{aligned}
z_{0} & =z_{v}^{\prime}, \\
T & =\frac{2}{c} \frac{z_{v}^{\prime}}{\cos \phi_{0}}=\frac{2}{c} \frac{z_{v}^{\prime}}{\cos \phi_{1}}, \\
D^{\prime} & =2 z_{0}^{\prime} \tan \phi_{0}=2 z_{0}^{\prime} \tan \phi_{1}, \text { and } \\
f & =f^{\prime} \cos \phi_{0}=f^{\prime} \cos \phi_{1},
\end{aligned}
$$

$\phi_{1}=$ angle of incidence of waves upon the ionosphere,

$\phi_{0}=$ half the vertex angle of the equivalent triangular path,

$z_{0}=$ height of equivalent triangular path,

$T^{\prime}=$ time the wave spends in the ionosphere, and

$D^{\prime}=$ horizontal distance the wave travels in the ionosphere.

We see that the relation $z_{v}=z_{v}{ }^{\prime}$ is valid in case (c) but not in any other case. The relation $T=\frac{2}{c} \frac{z_{v}^{\prime}}{\cos \phi_{1}}$ is valid in cases $\left(a^{\prime}\right)$ and $(b)$, but is not valid in general. The relation $D^{\prime}=2 z_{0}{ }^{\prime} \tan \phi_{1}$ holds approximately only in case $\left(a^{\prime}\right)$. And finally, $f=f^{\prime} \cos \phi_{0}$ in case $(c), f=f^{\prime}$

D. F. Martyn, Proc. Phys. Soc. (London) 47, 332 (1935). 
$\cos \phi_{1}$ in case $\left(a^{\prime}\right)$, and $f$ is a complicated function in every other case. It is therefore concluded that the equivalence theorem, in the form given, cannot be applied to the curved-earth problem.

Referring again to the summaries of cases $\left(a^{\prime}\right),(b)$, and $(c)$, we may for a given value of $z_{0}^{\prime}$ take a set of values of $z_{0}$, for each of which can be calculated the variation of $D$ with $\phi_{1}$, in each of the three cases. We may also, for these values of $z_{v}{ }^{\prime}$ and $z_{0}$, calculate the variation of $f^{\prime} / f$ with $\phi_{1}$. By eliminating $\phi_{1}$ graphically, we can determine the variation of $f^{\prime} / f$ with $D$ for a given $z_{v}^{\prime}$ and $z_{0}$, or, what is the same thing, the variation of $f^{\prime} / f$ with $z_{v}^{\prime}$ for a given $D$ and $z_{0}$. We can plot a family of transmission curves, with $f$ as abscissas and $z_{v}^{\prime}$ as ordinates, for each value of $D$ and several values of $z_{0}$, corresponding to each of these cases.

Such curves may be superimposed on the $\left(z_{v}{ }^{\prime}, f\right)$ curve obtained at vertical incidence, and the $\left(z_{0}, f\right)$ curve deduced therefrom, to give directly the wave frequency $f^{\prime}$ corresponding to reflection at a given level $z_{0}$, which is characterized by a given $z_{0}^{\prime}$. The value of $z_{0}$ appropriate to reflection at the given beight determines which curve of the family for a given $D$ is to be used, and the point of reflection is determined as the intersection of this transmission curve with the $\left(z_{0}^{\prime}, f\right)$ curve.

The value of $z_{v}$ depends only on $D$ and $\phi_{1}$, and so lines of equal $z_{v}$ may be plotted on the curve sheet for each $D$, so that $z_{v}$, as well as $f^{\prime}$, may be read off directly.

Because of the approximations made in case $(b)$ and the assumption of a linear variation of ionic density with height, this case is of only special significance. It will be assumed, until further investigation determines more precisely the variation of conditions with $z_{0}$, that the curves vary smoothly between those calculated for $z_{0}=0$ and those calculated for $z_{0}=z_{v}^{\prime}$.

A family of curves for each distance is rather cumbersome for rapid use. It is, as was said above, more convenient to use the log sec $\phi_{0}$ transmission curves, and apply a correction to the $\left(z_{v}^{\prime}, f\right)$ curve by multiplying each vertical-incidence frequency by the factor $1+\Delta f / f$, where

$$
1+\frac{\Delta f}{f}=\frac{\cos \phi_{0}}{\sqrt{1-\mu_{0}^{\prime 2}}} .
$$

This factor is obtained, for a given $D, z_{v}{ }^{\prime}$ and $z_{0}$, by determining corresponding values of $D$ and $\sqrt{1-\mu_{0}^{\prime 2}}$ for arbitrary values of $\phi_{0}$ or $\phi_{1}$. It is unity for $z_{0}^{\prime}=z_{0}$ and is quite easily obtained for $z_{0}=0$. For intermediate values of $z_{0}$ it will be assumed that the factor $1+\Delta f / f$ varies in a manner similar to that determined from the relation eq $7 \mathrm{~b}$ with $z_{v}=z_{v}^{\prime}$, i. e.,

$$
\frac{\cos \phi_{0}}{\sqrt{1-\mu_{0}^{\prime 2}}}=\sqrt{1-\frac{2\left(z_{0}^{\prime}-z_{0}\right)}{R+h} \tan ^{2} \phi_{0}},
$$

but drawn through the values for $z_{0}=0$ and $z_{0}=z_{v}{ }^{\prime}$ determined in the more precise analysis, rather than those indicated on the assumption that $z_{0}=z_{0}^{\prime}$. Cos $\phi_{0}$ is here calculated for an equivalent triangular path of height $z_{v}^{\prime}$. 
Figure 5 gives the approximate factors by which $f$ must be multiplied to give $f^{\prime} / \mathrm{sec} \phi_{0}$ for values of $z_{v}^{\prime}$ from 200 to $500 \mathrm{~km}$ and for distances up to $4,000 \mathrm{~km}$.

\section{EFFECT OF THE EARTH'S MAGNETIC FIELD}

The presence of the earth's magnetic field introduces some complications in the use of these transmission curves. These complications are often of minor importance compared with some of the unknown
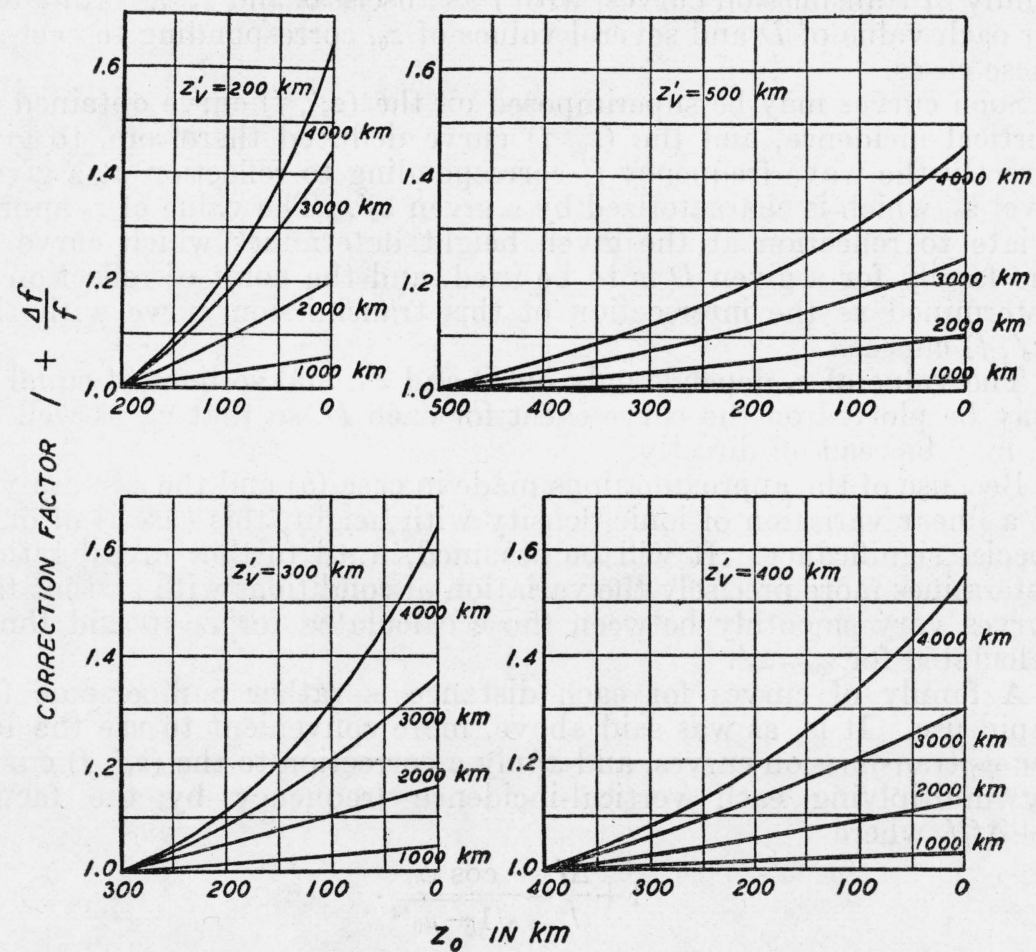

$z_{0}$ in km

FIGURE 5.-Approximate factors by which the equivalent vertical-incidence frequency $f$ must be multiplied to give the frequency $f^{\prime} / s e c \phi_{0}$ which is to be used in conjunction with the log sec $\phi_{0}$ curves.

factors (e. g., the geographic uniformity of the ionosphere over the transmission path), especially over long distances, but the effect of the earth's field must at times be taken into account. The anisotropy of the ionosphere due to this field causes the effect of the field on radio transmission to vary with the length, direction, and geographic location of the transmission path.

One effect of the field is to cause the received signal to be split in general into two main components, the one with the lower maximum usable frequency known as the " 0 " component and the other as the " $x$ " component. The refractive index for a frequency $f^{\prime}$, in the presence of a magnetic field $H$, whose components along and trans- 
verse to the direction of phase propagation are, respectively, $H_{\mathrm{L}}$ and $H_{\mathrm{T}}$ is given ${ }^{6}$ by

$$
\mu^{\prime 2}=1-\frac{f_{0}^{2}}{f^{\prime 2}-\frac{f^{\prime 2} f_{T}{ }^{2}}{2\left(f^{\prime 2}-f_{0}{ }^{2}\right)} \pm \sqrt{\left[\frac{f^{\prime 2} f_{T}{ }^{2}}{2\left(f^{\prime 2}-f_{0}^{2}\right)}\right]^{2}+f^{\prime 2} f_{L}{ }^{2}}},
$$

where

$$
\begin{aligned}
& f_{0}=\sqrt{\frac{\overline{N e^{2}}}{\pi m}}, \\
& f_{T}=\frac{e}{2 \pi m c} H_{T}, \\
& f_{L}=\frac{e}{2 \pi m c} H_{L}, \text { and } \\
& N=\text { ionization density. } \\
& \text { Upper sign refers to o-component; lower } \\
& \quad \text { to } x \text {-component. }
\end{aligned}
$$

The frequency of the wave whose $x$-component is returned from a given ionization density, at a given height, is different from the frequency of the wave whose o-component is returned from the same level. This frequency separation is in general a function both of frequency and distance and may of ten become negligible at great distances.

For practical calculation, it may be assumed that only the field and direction of wave propagation in the region near the level of reflection will appreciably affect the propagation of the wave. This assumption is probably better for the 0 - than for the $x$-component, and is, it must be emphasized, only a good approximation.

With this limitation, therefore, the value of $\mu^{\prime 2}$ may be calculated for a given transmission frequency and transmission path. If we put $\mu^{\prime}=\sin \phi_{0}$ and deduce $z_{v}$ from $\sin \phi_{0}$ as was done in the previous paper, we may plot transmission curves, of virtual height against equivalent vertical-incidence frequency, for the 0 - and $x$-components. When these curves are applied to the corrected $\left(z_{v}{ }^{\prime}, f\right)$ curves, they may be expected to give reasonably good results. An example of this type of transmission curve is shown in figure 6 . The one curve gives transmission conditions for the $x$-component, and the other for the 0 -component. The frequency used is well over the gyrofrequency $f_{\mathrm{B}}=$ $e H / 2 \pi m c$ so that the $x$-component is returned from a lower level than is the o-component and has a higher limiting frequency.

It is not now justifiable to plot the transmission curves logarithmically, since the form of the curves will vary with the transmission frequency $f^{\prime}$. For practical purposes, however, a logarithmic curve may be used within a limited range of wave frequencies about the frequency $f^{\prime}$ for which the curve is plotted; a practical limit might be, say, within \pm 15 percent of this frequency.

The logarithmic sec $\phi_{0}$ transmission curves may be used in estimating the maximum usable frequency for each component over a given path by adding or subtracting the separation between the limiting frequencies for the two components, evaluated at that frequency and distance. This separation is in general a function only of the trans-

6 E. V. Appleton. J. Inst. Elec. Engrs. (London), 71, 642 (1932). 
mission frequency and the quantity sec $\phi_{0}$, and may be estimated within the limits of experimental error in most cases.

Figure 7 gives the frequency to be added or subtracted from the maximum usable frequency given by the logarithmic sec $\phi_{0}$ transmission curves for the cases $H_{L}=0, H_{T} \doteq 0$, and $H_{T}=H_{L}$. The $H_{T}=0$ case is uninteresting save for single-hop transmission over the magnetic equator, close to the magnetic meridian. For transmission in the continental United States $H_{L}$ is much less than $H_{T}$ and, indeed, is negligible over east-west paths, so that such transmission we may consider as essentially transverse transmission. In this case,

$$
\mu^{\prime 2}=1-\frac{f_{0}^{2}}{f^{\prime 2}-\delta \frac{f^{\prime 2} f_{\mathrm{H}}{ }^{2}}{f^{\prime 2}-f_{0}{ }^{2}}},
$$

where $\delta=0$ for the 0 -component and 1 for the $x$-component, and the logarithmic sec $\phi_{0}$ transmission curves give the correct maximum

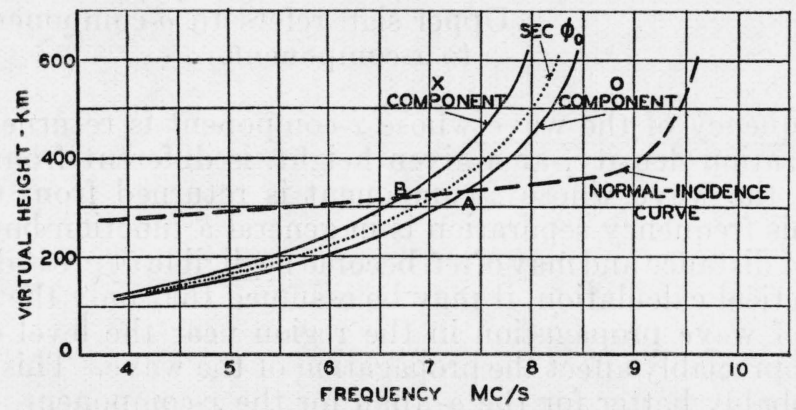

FIgURE 6.--Transmission curve, including effect of earth's magnetic field at level of reflection.

Dotted line =secant-law curve $\left(f^{\prime}=f\right.$ sec $\left.\phi_{0}\right)$ for the given distance $D$ and wave frequency $f^{\prime}$. Dashed line $\Rightarrow$ $\left(z^{\prime}, f\right)$ curve for the o-component, this being the component which, at vertical incidence is a measure of the ionization density $N$. The 0 -component of the frequency $f^{\prime}$ over the given distance is reflected at $A$, and the $x$-component at $B$.

usable frequency for the o-component. For this reason the o-component lies along the sec $\phi_{0}$ axis in the $H_{L}=0$ case in figure 7 .

For a maximum usable frequency for the 0 -component below the gyrofrequency $f_{H}=\sqrt{f_{T}{ }^{2}+f_{L}{ }^{2}}$ the $x$-component always has a maximum usable frequency above $f_{H}$. This is only important for $E$-layer transmission and only in cases where the $x$-component is not too highly absorbed at frequencies near $f_{H}$. This case must not be confused with the case of a transmission frequency $f^{\prime}$ less than $f_{H}$, in which case the $x$-component is reflected from a level above the level where the 0 -component is reflected.

Another effect of the anisotropy of the ionosphere due to the earth's field is to cause a difference in the directions of phase and energy propagation in the medium. This results in the wave's being reflected, not at the level where the direction of phase propagation is horizontal, but where the direction of energy flow (group direction) is horizontal. This effect has not been considered in these curves, and is probably not important to the degree of accuracy to which these calculations are carried. 

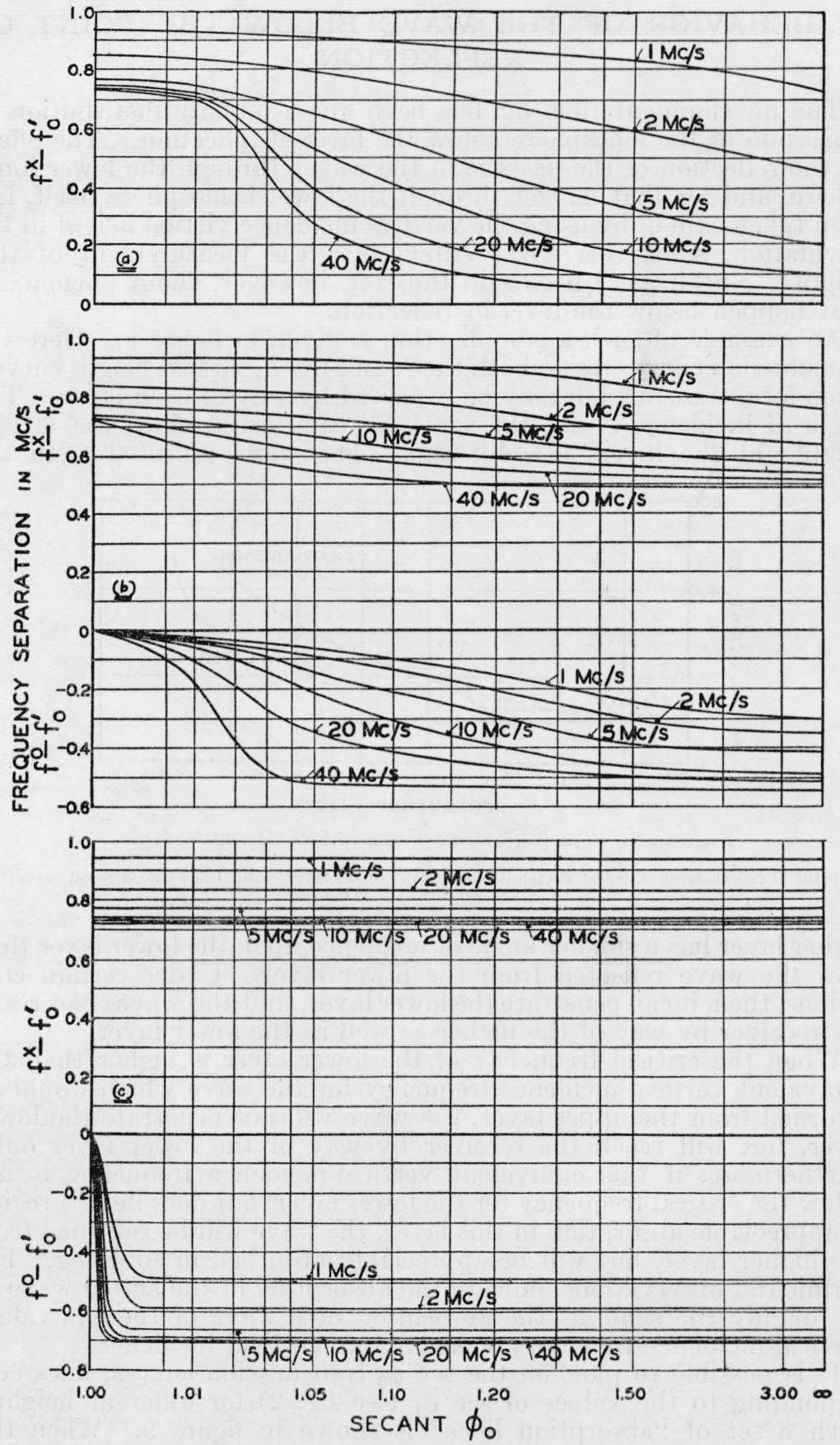

Figure 7. $f^{x}-f_{0}{ }^{\prime}$ and $f^{0}-f_{0}{ }^{\prime}$ plotted against sec $\phi_{0} f^{x}=$ frequency whose $x$-component is reflected at the same level as is the o-component of $f^{0}$.

$f_{0}^{\prime}=f^{\prime} \cos \phi_{0}$

The value of $f^{\prime}$ is given, in Mc/s, on each curve. Curves $(a) H_{L}=0$. Here $f^{0}-f_{0}^{\prime}=0$ and the curves for the 0 -component lie on the sec $\phi_{0}$ axis. Curves $(b) H_{L}=H_{T} \neq 0$. Curves $(c) H_{T} \doteq 0$. Here $f^{x}-f_{0}^{\prime}$ and $f_{0}-f_{\varepsilon}^{\prime}$ are independent of sec $\phi_{0}$, save near sec $\phi_{0}=1$. 


\section{BEHAVIOR OF THE WAVE BELOW THE POINT OF REFLECTION}

The development thus far has been given for any distribution of ionization in the ionosphere below the level of reflection. The effect on the reflection of the passage of the waves through the lower ionosphere, and for that matter through the lower atmosphere itself, has been taken care of by using the vertical incidence virtual height in the calculation, since this effect enters into the measurement of this height. Nothing has been said thus far, however, about phenomena that happen below the level of reflection.

An example of such a complication is given in figure 8 . Here the transmission curve crosses both the $E$ and the $F_{2}$ virtual-height curves. Therefore, transmission may be expected by way of each layer. The angle of incidence is not the same for each layer, since the virtual heights of the layers are different. The wave reflected from the

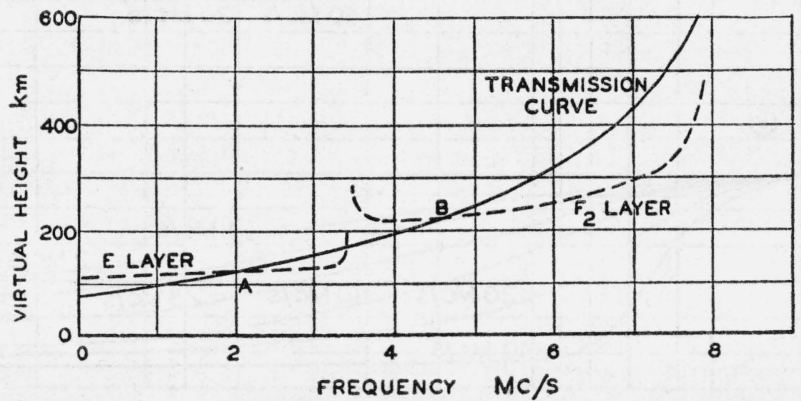

FiguRe 8.-Transmission by way of both $E$ and $F_{2}$ layers.

$A=$ point of reflection on $E$ layer; $B=$ point of reflection on $F_{2}$ layer; solid line=transmission curve; and dashed line $=\left(z_{\imath}{ }^{\prime}, f\right)$ curve.

higher layer has a smaller angle of incidence upon the lower layer than does the wave reflected from the lower layer. Under certain conditions, then, it can penetrate the lower layer, and the waves can reach the receiver by way of the higher as well as the lower layer.

When the critical frequency of the lower layer is higher than the equivalent vertical-incidence frequency for the wave which would be returned from the upper layer, the wave will not penetrate the lower layer, but will reach the receiver by way of the lower layer only. Furthermore, if this equivalent vertical-incidence frequency is not below the critical frequency for the lower layer, but does lie in a region of appreciable absorption in this layer, the wave will be returned from the higher layer, but will be appreciably absorbed in so doing. Experimental observations indicate the absorption of the actual wave to be roughly the same as the absorption of a wave of the equivalent vertical-incidence frequency measured at vertical incidence.

It is possible to plot, on the sec $\phi_{0}$ transmission curves, lines corresponding to the values of sec $\phi_{1}$ (see fig. 2) for different heights. Such a set of "absorption lines" is shown in figure 9. When the transmission curve is superimposed on the $\left(z_{0}^{\prime}, f\right)$ curve the behavior of a wave below the reflection level may be estimated by the region of the $\left(z_{v}^{\prime}, f\right)$ curve sheet through which the absorption line through the reflection point passes. If this line passes through a region of 
absorption or cuts a lower layer the wave will be absorbed or will not penetrate through to the higher layer.

These absorption lines are the lines sec $\phi_{1}=$ const. for a plane earth and for short distances on a curved earth. They curve toward larger

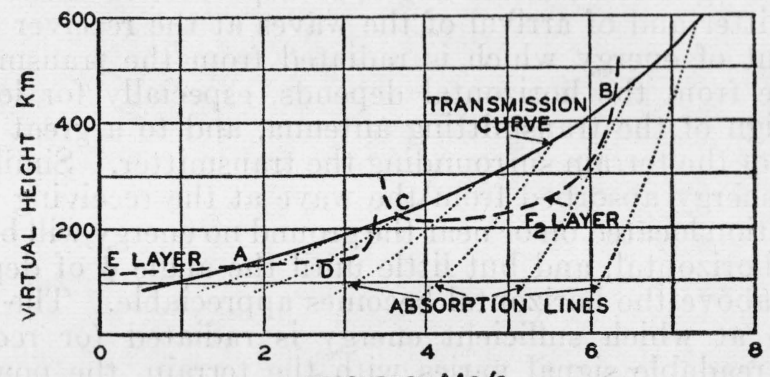

FREQUENCY MC/S

FigURE 9.-Absorption lines on logarithmic transmission curve.

Dashed line $=\left(z_{0}{ }^{\prime}, f\right)$ curve; dotted lines=absorption lines. $E$ reflection takes place at point $A$ and $F_{2}$ reflection at point $B . \quad F_{2}$ reflection at $C$ is shielded by the $E$ layer at $D$.

values of sec $\phi_{1}$ for lower heights in the case of greater distances over a curved earth, and approach the transmission curve itself for great distances. An example of the use of the transmission curves and
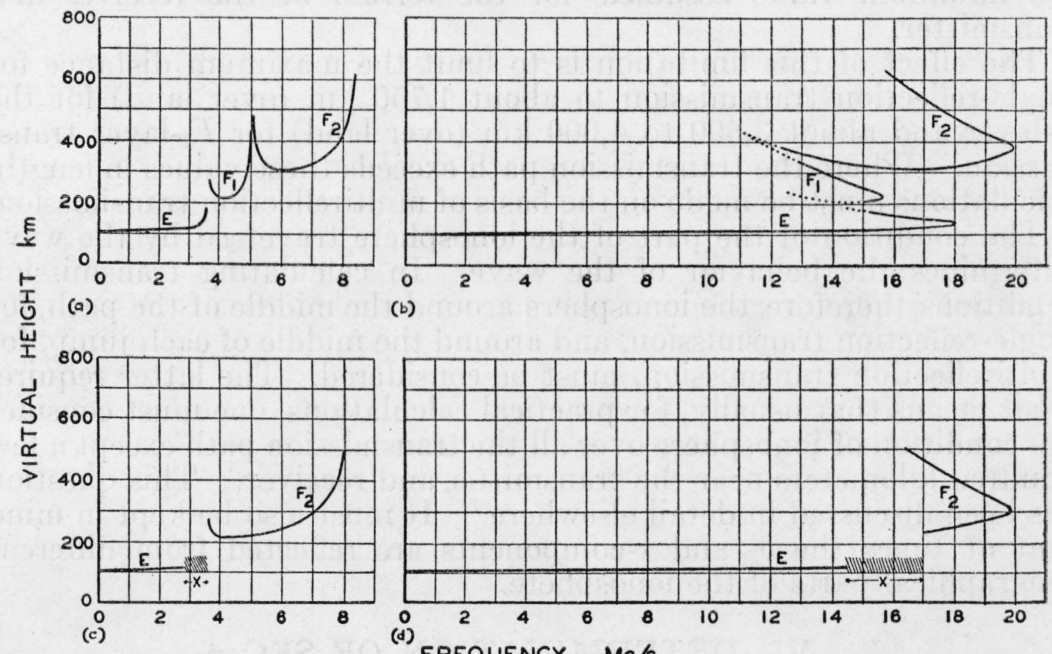

(b)

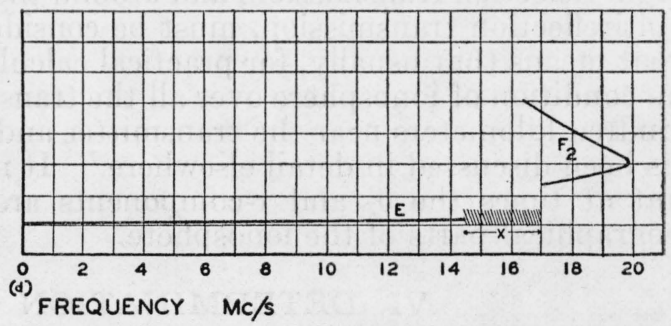

FIGURE 10.-- irtual heights at vertical and oblique incidence.

(a) $\left(z_{v^{\prime}}^{\prime}, f\right)$ curve showing $E, \mathrm{~F}_{1}$, and $F_{2}$ layers. (b) oblique-incidence heights corresponding to $(a)$. Dotted lines show parts of curves shielded by $E$ layer. Note transmission of some frequencies by several layers. This shows how the $F_{1}$-layer reflections are relatively unimportant for transmission. $(c)\left(z z_{0}^{\prime}, f\right)$ curve showing absorption $(X)$ above critical frequency for the $E$ layer. $(d)$ oblique-incidence heights corresponding to $(c)$. Absorption here $(X)$ completely blocks out some frequencies. All curves are for the o-component.

absorption lines is shown in figure 10, which gives vertical-incidence curves and the corresponding frequency-height curves derived therefrom for the transmitted wave over a distance. From these latter curves can be deduced, of course, the equivalent paths or group retardation of the waves over the transmission path. 


\section{ANGLE OF DEPARTURE AND ARRIVAL OF THE WAVES}

Disregarding any possible asymmetry of the wave trajectory due to the earth's magnetic field, the angles of departure of the waves from the transmitter and of arrival of the waves at the receiver are equal. The amount of energy which is radiated from the transmitter at a given angle from the horizontal depends, especially for low angles, on the design of the transmitting antenna, and to a great extent on the nature of the terrain surrounding the transmitter. Similar factors affect the energy absorbed from the wave at the receiving station.

For a station located on or near the ground no energy will be radiated below the horizontal, and but little until the angle $\psi$ of departure of the waves above the horizontal becomes appreciable. The minimum value of $\psi$ at which sufficient energy is radiated (or received) to produce a readable signal varies with the terrain, the power of the transmitter and the sensitivity of the receiver. Over sea water $\psi$ can be very nearly zero; over land the minimum value may be several degrees. A fair average approximation may be that $\psi$ must exceed about $3.5^{\circ}$.

A simple geometrical calculation gives $\psi$ in terms of $\sec \phi_{0}$ for various distances, and these may be noted on the transmission curves. The point of reflection must then correspond to an angle $\psi$ greater than the minimum value assumed for the terrain at the receiver and transmitter.

The effect of this limitation is to limit the maximum distance for single-reflection transmission to about $1,750 \mathrm{~km}$ (over land) for the $E$-layer and about 3,500 to $4,000 \mathrm{~km}$ (over land) for $F_{2}$-layer transmission. Where the transmission path exceeds these values in length, calculations must be made on the basis of multireflection transmission.

The condition of the part of the ionosphere traversed by the wave determines the behavior of the wave. In calculating transmission conditions, therefore, the ionosphere around the middle of the path, for single-reflection transmission, and around the middle of each jump, for multireflection transmission, must be considered. The latter requirement means that usually, for practical calculations, one must consider the condition of ionosphere over all the transmission path except a few hundred kilometers near the transmitter and receiver. This question has been discussed in detail elsewhere. ${ }^{7}$ It must also be kept in mind that at times the 0 - and $x$-components are reflected from different geographical parts of the ionosphere.

\section{DETERMINATION OF SEC $\phi_{0}$}

Figure 11 gives an alignment chart for the rapid determination of the factor sec $\phi_{0}$ to be used in calculating the logarithmic transmission curves. To use the chart, place a straightedge so that it passes through the desired virtual height and the desired distance laid off on the distance scale at the lower left-hand edge of the chart (increasing distances lie to the left). The ordinate of the intersection of the straightedge with the vertical line corresponding to the same

${ }_{7}$ T. R. Gilliland, S. S. Kirby, N. Smith, and S. E. Reymer. Characteristics of the ionosphere and their applications to radio transmission. J. Research NBS 18, 645 (1937) RP1001; Proc. Inst. Radio Engrs. 25, 823 (1937)

The weekly radio broadcasts of the National Bureau of Standards on the ionosphereand radio-transmission conditions. Letter Circular LC499. 


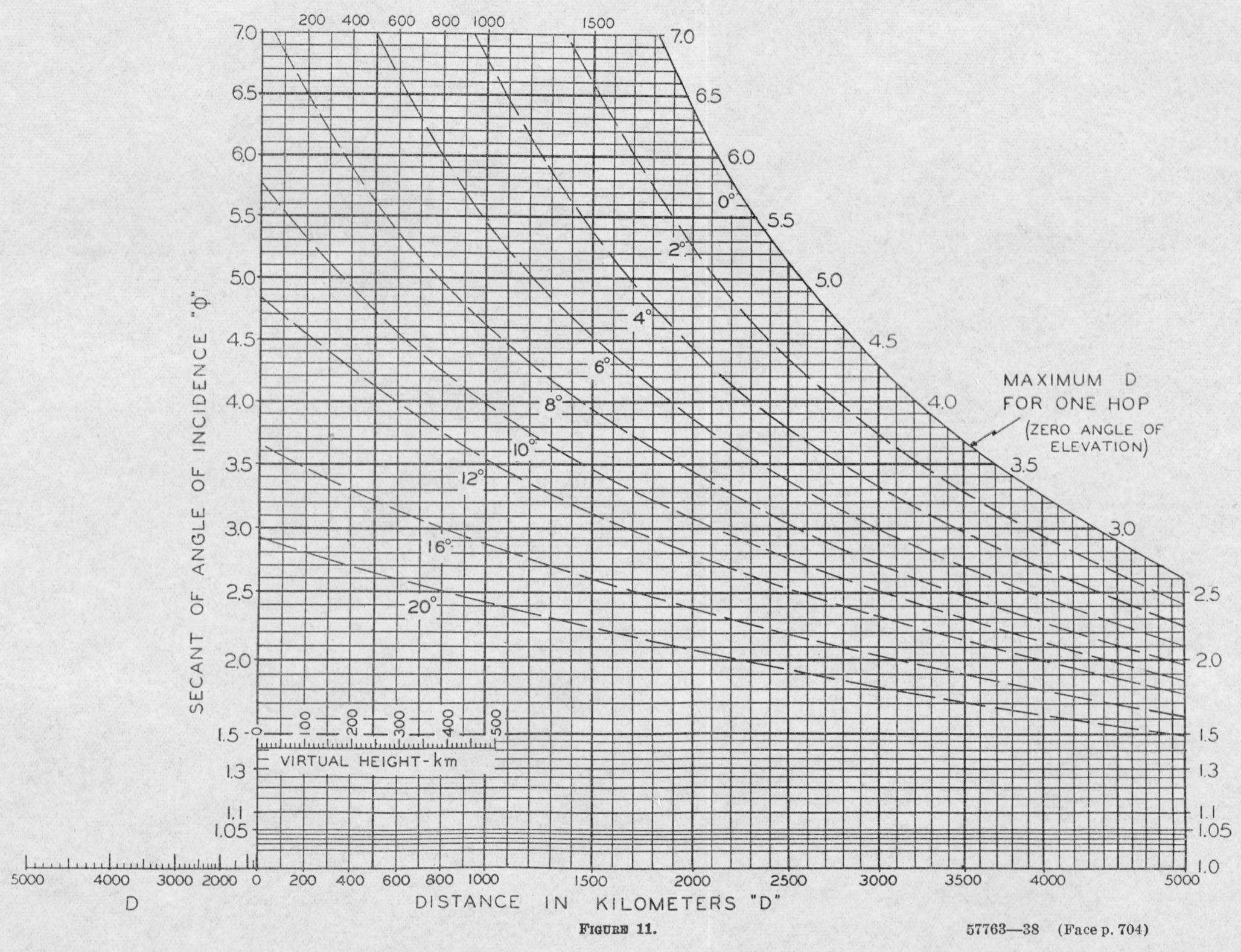


desired distance laid off on the main distance scale (increasing distances lie to the right) gives the value of $\sec \phi_{0}$. The relation of the point of intersection to the curved dashed lines of equal $\psi$ gives the value of the angle of departure of the waves from the horizontal. A point of intersection falling above the $\psi=0^{\circ}$ line indicates an impossible case, where the ray would have to depart at an angle below the horizon.

For example, a distance of $2,400 \mathrm{~km}$ and a virtual height of 300 $\mathrm{km}$ corresponds to a sec $\phi_{0}$ of 3.07 , and an angle of departure of $8.2^{\circ}$.

\section{TRANSMISSION FACTORS}

When average transmission conditions over a period of time or when a variety of transmission paths are to be considered, or when an estimate of the maximum usable frequencies is to be made without a precise knowledge of the ionosphere over the transmission path, it is convenient to have available a means by which the maximum usable frequencies may be quickly estimated from an approximate value of the vertical-incidence critical frequency.

The National Bureau of Standards is now beginning a compilation of factors by which the critical frequency for the o-component, measured at vertical incidence, may be multiplied in order to obtain the maximum usable frequencies. These factors are based on average observations over a period of time, and may be applied either to average critical frequencies to give average transmission conditions, or to a given observation of a critical frequency to obtain approximate transmission conditions at a given time.

\section{CONCLUSIONS}

The type of transmission curves described above have been in use at the National Bureau of Standards for the past 2 years in studying the correlation of high-frequency radio-transmission conditions with regular ionosphere observations. The results of continuous measurements of high-frequency broadcasting stations and observations on other high-frequency signals, as well as the results of some specific experiments have been compared with verticalincidence data. Practically all the available data agree with what would be expected on the basis of the theory outlined above, and the exceptions may in most cases be accounted for.

Washington, November 22, 1937. 